\title{
Social Middleware for Civic Engagement
}

\author{
Rafael Angarita \\ ISEP Paris, France \\ Email: rafael.angarita@isep.fr
}

\author{
Nikolaos Georgantas \\ Inria Paris, France \\ Email: Nikolaos.Georgantas@inria.fr
}

\author{
Valérie Issarny \\ Inria Paris, France \\ Email: Valerie.Issarny@inria.fr
}

\begin{abstract}
Civic engagement refers to any collective action towards the identification and solving of public issues. Current civic technologies are traditional Web- or mobile-based platforms that make difficult, or just impossible, the participation of citizens via different communication technologies. Moreover, connected objects sensing physical-world data can nourish participatory processes by providing physical evidence to citizens; however, leveraging these data is not direct and still a time-consuming process for civic technologies developers. This paper introduces the concept of social middleware for civic engagement. Social middleware allows citizens to engage in participatory processes supported by civic technologies- via their favorite communication tools, and to interact not only with other citizens but also with relevant connected objects and software platforms. The mission of social middleware goes beyond the connection of all these heterogeneous entities. It aims at easing the implementation of distributed applications oriented toward civic engagement by featuring dedicated built-in services.
\end{abstract}

Index Terms-Citizen-sensor-networks, Citizen empowerment, Civic IoT, Civic technology, Middleware, Cyber-Physical-Social Systems

\section{INTRODUCTION}

Civic engagement, as defined by the American Psychological Association", is any "individual and collective action designed to identify and address issues of public concern." It is all about caring and working to address issues that concern a community, from a local to a global scale. Citizens engage by collectively bringing new ideas and solutions, sharing those ideas and discussing and improving them, choosing the ones they prefer and putting them in action. Examples of civic engagement are numerous. They range from reporting wild water storage that contributes to the proliferation of mosquitoes, building maps of marginalized urban areas, deciding what to do with a budget allocated to a local community, to campaigning and voting in national general elections.

Pervasive digital technologies are considered to be a significant enabler of civic engagement. They pave the way for more direct democratic processes by facilitating the online, direct participation/engagement of people in government/community actions. Further, the design of the supporting socially-oriented digital technologies has become a major research topic following the mass adoption of social networking. The research in the area is broad, and is often multi-disciplinary, crossdisciplinary and even trans-disciplinary [1], [2]. The research in the digital sciences spans the full software stack, from networking up to user interfaces. This includes middlewarerelated research, which is primarily concerned with either

\footnotetext{
${ }^{1}$ http://www.apa.org/education/undergrad/civic-engagement.aspx
}

analyzing social relations across social networks [3], [4], [5] or enforcing privacy [6], [7], [8].

Despite the above trends, digital technologies supporting inclusive participation toward civic engagement remains a major challenge -even at a local scale. Understanding the motivations for public engagement has been and is still the focus of various studies, especially in the HCI domain (e.g., [9], [10], [11], [12], [13], [14], [15], [16]). However, studies concentrate on very specific use cases. Similarly, supporting digital technologies result in a specific function or platform, which limits their impact with respect to fostering massive scale engagement. It is our vision that middleware has a significant role to play. We argue that social middleware is the necessary building block to enable and foster interactions among the many relevant "civic actors" in a way that does not prevent participation of diverse actors due to the use of heterogeneous and distributed technologies, but rather reconciles them. Our work shares consideration with the Internet of People vision that revisits the Internet to integrate people and their devices as first class network entities [17]. We revisit the middleware paradigm so that it integrates interaction with people and technical objects.

We define social middleware as middleware focused on both (1) allowing people to interact for the sake of civic engagement, and (2) enhancing the quality of participation. To this end, social middleware must support:

- Civic social networking. Social middleware lets people interact, whatever technologies they use. Social middleware also accounts for the blending of the physical and virtual worlds in which connected objects and software platforms are vehicles that allow informing but also enact civic engagement (e.g., using public displays to gather and share information, leveraging crowdsensing to map environmental issues). Ultimately, interactions mediated by social middleware, whether between humans, between humans and machines, or between machines are all human-centered, building upon online social network services.

- Incentives to enhance civic engagement. The goal of social middleware is to enhance the quality of civic engagement. Accordingly, since it coordinates civic technologies and citizens, social middleware can support additional incentives to those provided by the underlying civic technologies. Incentives can be in the form of recognition of citizens for their participation or by 
gamifying the participation process within the particular technologies citizens use. Further, every actor (spanning individuals, communities, governments but also platforms and connected objects) involved in social middleware ecosystems must adhere to a set of data privacy and security policies that ensure ethical participation. For instance, complying to the GDPR that is now enforced by $\mathrm{EC}$ is one obvious requirement.

The paper makes the following contributions towards the design of social middleware:

- Section II surveys the diverse digital technologies enabling online civic engagement, and the related literature, from which we derive the essential requirements for social middleware.

- Section III introduces the key concepts underlying social middleware, that is, enabling civic social networks across the many relevant civic actors that include citizens but also digital services associated with software platforms and the Internet of Things.

- Section IV introduces the social middleware bot (sm-bot) concept, which features social bots that enable sociallydriven interactions among the relevant civic actors. To do so, sm-bots overcome the heterogeneity of the underlying digital platforms and implement socially-enhanced interactions as promoted by online social networks.

- Further to enabling socially-driven interactions among the many relevant actors, the social middleware features functions dedicated to fostering the engagement of the target actors, while dealing with security and privacy. Section V surveys such functions where we build upon the civic technologies state of the art.

Finally, Section VI concludes with our ongoing and future work towards the implementation of the social middleware solution.

\section{TeChnologies EnABling ONLINE CIVIC ENGAGEMENT}

There is no doubt that digital technologies have changed, and will keep changing, the fabric of society. They have transformed the way humans interact, consume information and, as a consequence, influence the physical world. This especially holds for civic engagement for which digital technologies hold the promise of sustaining engagement at a very large scale [18].

\section{A. Social media, social networks, and messaging applications}

Social media allow people to easily share their ideas, debate with others, organize, collaborate and stay in contact. It is no surprise that social media increasingly play a major role in civic engagement, as they support the growth of large digital networks of activists, on the technological as well as on the economic level [19]. Two iconic instances where millions of people organized and mobilized using social media are the Arab Spring revolutions and the Occupy movement [20]. In the Arab Spring revolutions, activists used Facebook and text messaging to share reports on the events in the streets, while Twitter played a vital role in the transnational communication on these revolutions. By reporting on the demonstrations using major social media platforms such as Twitter, YouTube and Facebook, activists of the Occupy movement created a sense of the collective [21].

Along with social media, messaging applications such as email, SMS, WhatsApp, Telegram and Messenger play an important role in engaging and mobilizing citizens. For example, researchers showed that text message reminders via SMS produce a statistically significant increase in the likelihood of voting [22].

Despite the plethora of social media and messaging application offers, people using a particular platform cannot easily interact with people using a different one. To overcome this limitation, we have proposed USNB to enable users to meet and interact beyond the boundary of their particular communication technologies [23]. USNB is a middleware that enables interoperability across heterogeneous and distributed social media as well as messaging applications, to achieve "social interoperability".

Still, supporting civic engagement at scale, from informing to empowering to organizing, remains a challenge, which Civic Technologies aim at tackling.

\section{B. Civic technologies}

In contrast to general-purpose social media, civic technologies (aka Civic Tech) are conceived with the specific purpose of supporting civic engagement. They aim at enhancing the relationships between citizens and their governments by providing mechanisms to improve collaborative actions and participation in public decisions.

Various Civic Tech platforms have emerged over the last decade. They may be classified according to the key function they support [24]: (1) connecting \& informing to communicate with the citizens, (2) contributing $\&$ acting to empower people, and (3) organizing \& managing to change the way institutions work. Social media often serve the first function. A wellknown example belonging to the second category is Make.org (https://make.org) that is a petition platform enabling users to launch campaigns and collect signatures. Most platforms of the third category adopt the software as a service paradigm. One well-known example is NationBuilder (https://nationbuilder. $\mathrm{com} /$ ) that is dedicated to the management of election campaigns.

In the above context, we have introduced the AppCivist service-oriented platform so as to enable the assembly of thirdparty Civic Tech services that are the most relevant to support a given civic action [25]. AppCivist enables the configuration of workflows oriented toward citizen participation, which consist of all or part of the following set of operations: proposal making, collaborative proposal editing, deliberation, voting, and implementation. All the operations are in particular leveraged in the AppCivist-PB customization of the platform, which focuses on enabling the online participation to participatory budgeting campaigns [26]. 
With the exception of AppCivist, most Civic Tech platforms concentrate on one function of civic engagement, thereby leaving their smooth inter-working up to the end-users. Further, studies show that citizens must be provided with adequate data access [13], including leveraging appropriate data sources [27], [14]. This in particular calls for adequate connection with the physical world.

\section{IoT \& CPSS}

We believe that the Internet of Things (IoT) has a significant role to play in Civic Engagement. By blending the physical and virtual worlds, the IoT enables aggregating knowledge that informs citizens' actions. Further considering the IoT ability to sense and act upon the physical world, it creates new interaction opportunities for the citizens.

Mobile phone sensing illustrates well the added-value of the IoT to civic engagement. Citizens are increasingly adopting participatory sensing technologies as they allow them to collect and share data about the environment [28], [29], [30]. The emergence of CPSS -Cyber-Physical-Social-Systems- is another major illustration of the role that the IoT is playing in the development of social systems in general, and civic engagement in particular. CPSS stresses the human-centric vision for computing systems, which must provide contextually relevant abstractions to people out of the knowledge gathered from the virtual, physical and social worlds [31], [32].

This trend is questioning the design of the overall stack of Internet-based computing. The Internet of People vision in particular stresses the need to revisit the current Internet paradigm where people and their devices become active elements of the Internet and today's Internet becomes its core [17]. We share this vision, for which we advocate that a social middleware is sorely missing. Social middleware will provide the abstractions associated with these diverse "social entities" and their interactions, together with key functions supporting engagement at scale. This further raises the question of proper interactions with such social entities.

\section{Chat, Chatter \& Social bots}

Social bots, an evolution of the early software robots (aka bots) as part of the planetary-scale social networking, appears as a powerful technology solution to address interaction with social entities. A social bot is a type of conversational social agent that can interact with humans via any digital technology. Social bots are developed and deployed with specific goals by software engineers. For example, a Twitter social bot can twit a predefined message with a corresponding hashtag; a Whatsapp social bot can spam users with a message promoting a product; or a Facebook social bot can add random people as friends to later expose them to some content. Moreover, in the last years we have seen a rapid increment of social bots for automated customer service.

Because they impersonate humans in a way that may be un-noticed, social bots may be quite harmful [33]. Still, social bots may also work for the greater good. For example, Crisis Text Line (www.crisistextline.org) is a Facebook Messenger and SMS social bot offering a hotline for people experiencing mental health crises. It asks people the details of the crises, before handing the control of the conversation to a trained human counselor. Another example is that of early adopter nonprofits ${ }^{2}$, which have used bots to automate civic engagement, such as helping citizens register to vote, contact their elected officials, and elevate marginalized voices and issues.

For better or for worse, social bots thrive in political and civic engagement scenarios [34]. For example, Resistbot (resist.bot) is a social bot that lets anyone turn a text message into a fax to their representatives and senators. As of July 2017, more than 4 million faxes have been sent using the platform. Resistbot is present on Facebook Messenger, Twitter, Telegram and SMS. Social bots are increasing their presence in social network sites and messaging applications, since they have the potential of reaching millions of people and especially young people who otherwise would remain apathetic to civic engagement. For example, RockEnrol-bot (www.rockenrol.org.nz) is a social bot created by a digital community in New Zealand whose mission is to inform and engage young voters.

We cannot ignore social bots in the development of civic engagement applications and they may indeed support and foster civic engagement. However, they must behave in a ethical way; to ensure this as well as leverage all the capabilities for civic engagement offered by the technologies surveyed in this section, we advocate that a middleware solution is essential.

\section{The Social Middleware Vision}

Social middleware builds upon the notion of civic social network that establishes a collaborative framework for civic engagement. The term has been coined by others such as in [35] to emphasize the need to customize collaborative frameworks for the sake of civic engagement. However, to the best of our knowledge, existing work is mainly focused on developing specific Civic Tech platforms for the collaborative production of urban knowledge.

Social middleware embraces a broader vision of civic social networks, that is, fostering collaboration among the relevant entities of a CPSS to engage in a collective action so as to solve some public issue. Social middleware introduces a set of abstractions that address the engagement and coordination of civic actors. Such abstractions can be leveraged by civicoriented applications. Going one step further, our definition of civic actors spans citizens, online digital services, and things liaising with the physical world. In particular, and in accordance with the general idea of the Actor-Network Theory [36], which states that human and non-human actors should receive equal treatment and be described in the same terms, an actor involved in a civic-oriented application may be any relevant entity capable of sending and/or receiving data. It does not matter its language, the middleware protocol or syntax it uses, the knowledge it represents or its nature. All that counts is that an actor has an available Internet-based interface allowing interaction within the digital world.

${ }^{2}$ ssir.org/articles/entry/leveraging_the_power_of_bots_for_civil_society 


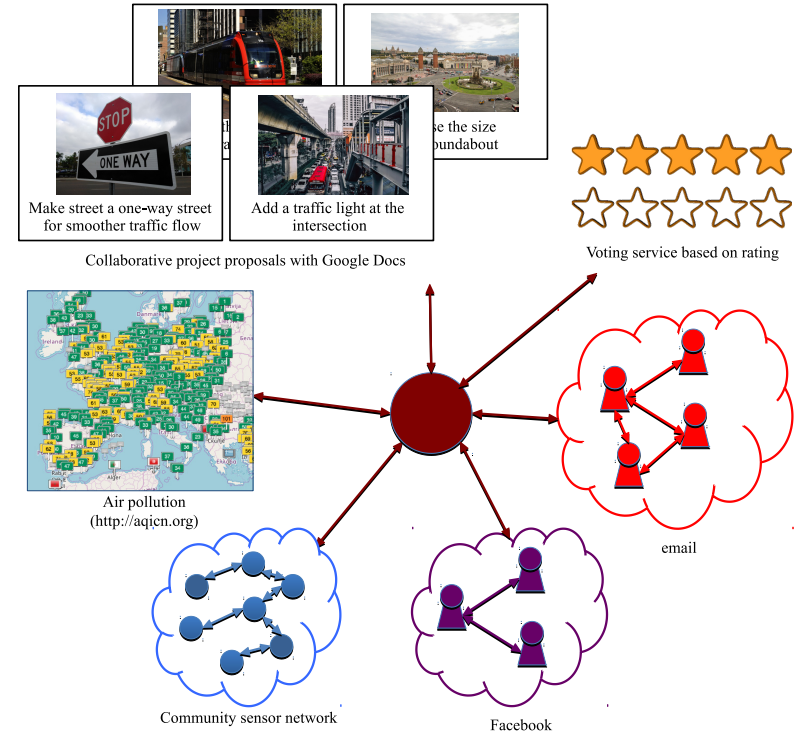

Fig. 1: A CPSS enabling a public consultation.

For illustration, we consider a public consultation about the implementation of measures to reduce the population exposure to the urban environmental pollution. A government or agency typically initiates and manages the consultation; it defines the timeline as well as presents the measures under consideration. The goal is to gather ideas and feedback from citizens. The participation may additionally benefit from thirdparty environmental knowledge such as participatory sensing applications (e.g., see [29]). Further, citizens may want to be able to exchange about the measures that the government/agency envisions, bringing in their perspective as much as asking questions to know more. In this public consultation, citizens can elaborate proposals collaboratively and/or vote for them and visualize the voting results.

As Figure 1 illustrates, the public consultation builds a civic social network that leverages services from existing technology platforms: popular social networks and simpler communication means such as email allow the citizens to get informed and interact, a web-based text editor enables collaborative proposal editing, a crowdsensing and a pollution monitoring service provide access to observations about the environmental pollution, an advanced visualization tool offers ways to map and visualize the available knowledge, and an existing online voting service enables gathering the citizens' votes.

Social middleware introduces the sm-bot concept to bridge seamlessly the civic actors that populate the above thirdparty technology platforms, while fostering civic engagement as part of civic-oriented applications. That is, sm-bots serve creating a civic social network overlay. The sm-bot is the core middleware component that implements built-in functions for socially-enhanced interoperability within digital platforms, as well as for supporting civic-engagement. The implementation of a civic-oriented application then relies on customizing smbots according to the specifics of the application, and deploy- ing the instances of the sm-bots in the relevant platforms. In practice and as depicted in Figure 2, the coordination of sm-bots as part of a civic-oriented application generates the coordination of the civic actors that are connected through the underlying platforms.

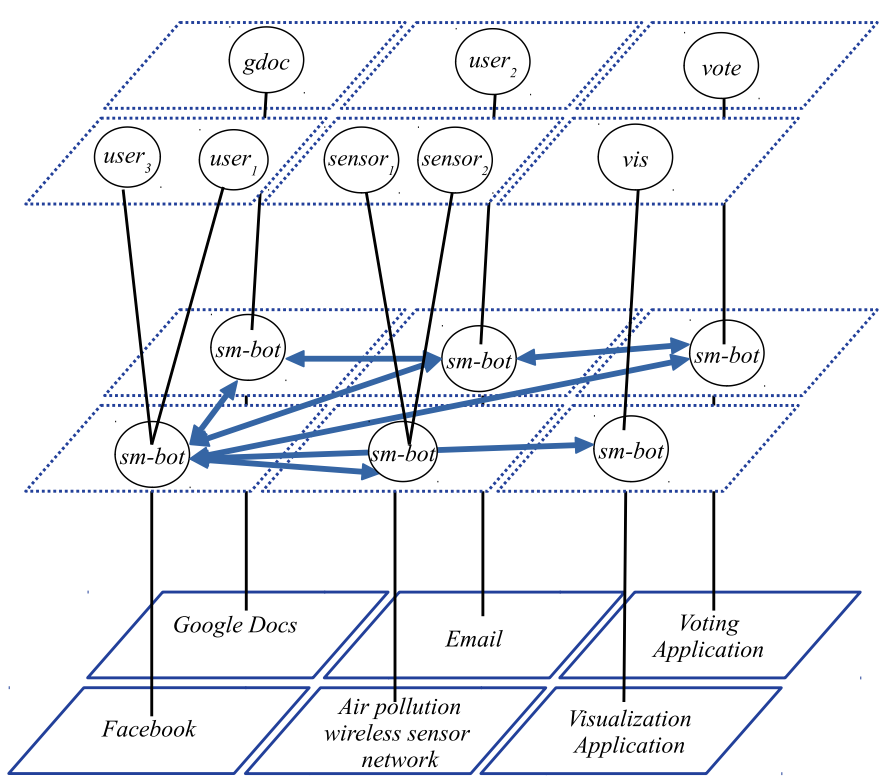

Fig. 2: Social middleware enacts civic social networks through the sm-bot abstraction that bridges technology platforms enabling online civic engagement.

\section{Sm-bots Enacting Civic Social Networks}

Social middleware brings to applications the abstraction of civic actor so as to ease the coordination of people as well as of connected digital entities toward civic engagement. As we survey in Section II, the online involvement of people within civic actions is primarily via Online Social Network Services. In particular, most civic technologies rely on some OSNS for user interactions. However, we argue that social middleware must enable people to interact across heterogeneous OSNSs so that each individual may use the most convenient tool, and thereby prevent the well-known platform lock-in issue.

\section{A. Interoperability across Online Social Networks}

Social middleware builds upon our previous work on the Universal Social Network Bus (USNB) ${ }^{3}$ enabling users to interact across OSNSs, where an OSNS may range from a base email platform to a full-fledged online social network supporting various social interaction services such as direct messaging or indirect wall posting [23], [37].

Figure 3 illustrates how USNB enables social interactions among four users of four different OSNSs, through the implementation of a multi-party, distributed mediator [38]. The (multi-party) USNB mediator embeds a USNB persona $p_{x}$ for each of the OSNSs $\Omega_{x}$ to connect them within the "universal social network". The personae are the main components of

${ }^{3}$ https://gitlab.inria.fr/usnb/ 


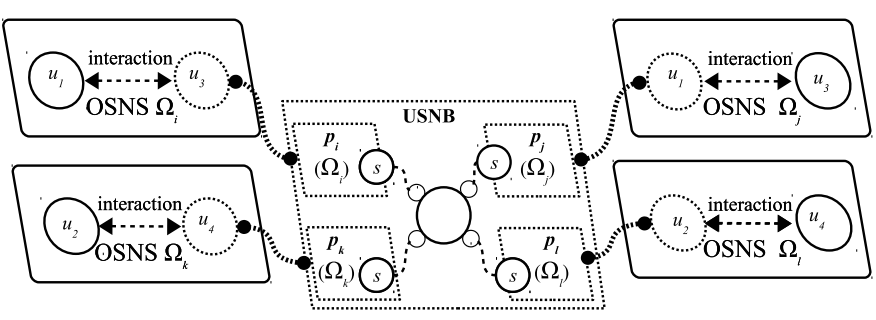

Fig. 3: The USNB multi-party distributed mediation across online social network services.

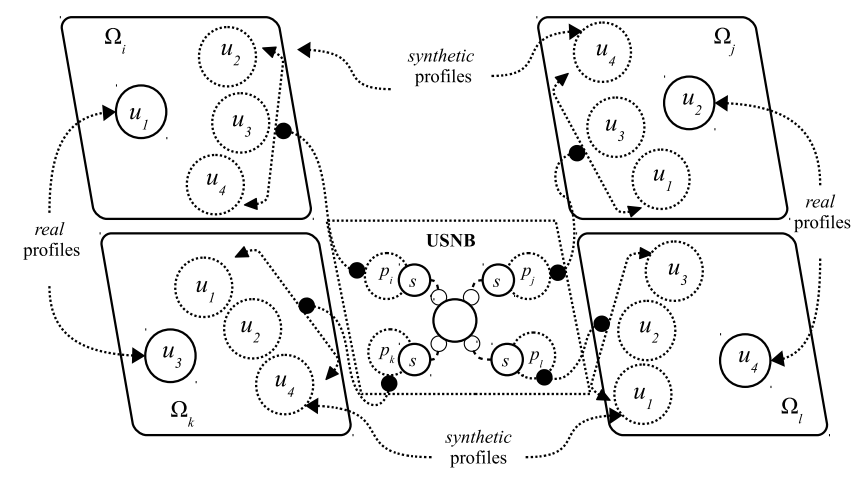

Fig. 4: Federating heterogeneous OSNSs via USNB and synthetic profiles (depicted using dotted circles).

USNB and they interact with each other through the USNB reference social interaction service $s$, that is, a persona implements the conversion logic between the OSNS's social interaction service and the USNB's reference social interaction service.

The linking of USNB personae allows users to interact independently of the OSNS they each belong to, including sharing their profiles across the OSNSs. Figure 4 illustrates the case of four users: $u_{1,2,3,4}$ of the OSNSs $\Omega_{i, j, k, l}$, respectively. USNB creates synthetic profiles for each of the users in all the OSNSs where they do not have a profile. For example, $u_{1}$ has a profile in $\Omega_{i}$, while USNB creates synthetic profiles for $u_{1}$ in $\Omega_{j}, \Omega_{k}$ and $\Omega_{l}$. The synthetic profiles, which are represented as dotted circles in the figure, enable (remote) users of distinct OSNSs to interact via the USNB personae. As a result, all the users in the USNB federation of heterogeneous OSNSs can interact. For example, $u_{1}$ and $u_{4}$ are connected and may interact, while the interactions in USNB are transparent for both of them: From $u_{1}$ 's perspective, interactions happen only via $\Omega_{i}$; from $u_{4}$ 's perspective, only via $\Omega_{l}$; in effect, $u_{1}$ and $u_{4}$ interact with synthetic profiles managed by the personae $p_{i}$ and $p_{l}$.

The sm-bot inherits from the USNB persona concept regarding the embedded support for interoperability across online social network services, and thereby enables the creation of a civic social network overlay over existing OSNSs. As a result, the sm-bot implements a reference social interaction service, which derives from the one of USNB and relates to ([23]): profile management and social interactions.

\section{B. Socially-enhanced Interactions with Civic Actors}

In addition to leveraging online social networking, civicoriented applications depend on diverse civic technologies to support the involved actions, such as informing, organizing, managing, etc. Also, the IoT is a valuable network to gather information from and act upon the physical world. Hence, social middleware creates a civic social network overlay over OSNSs complemented with relevant civic tech platforms and IoT networks. Still, a key challenge is how to support the interactions with these diverse digital entities as part of a civic social network. We argue that these interactions should be socially-enhanced, that is, adaptive to the interaction context and particularly to the civic actors -people, software services, things- that are involved.

Socially-enhancing the interactions with digital entities closely relate to the vision of "speaking objects" [39], where objects are capable of understanding different contexts in order to communicate in an appropriate and conversational way, as opposed to just transmitting and receiving raw data.

We highlight the following considerations associated with the sm-bot support for socially-enhanced interactions:

- Actor type and characteristics: The absence of nonverbal cues during social interactions in the virtual sphere can prevent participants from detecting demographic, personality, and interpersonal characteristics of others. According to [40], such an absence of nonverbal cues reduces the capacity of persuasion. Even though this study focuses on human-to-human interactions in computer-mediated communication, it informs how to foster meaningful interactions among all kind of actors when concerned with persuasion and engagement. Additionally and according to research on theories of computer-mediated communication [41], [42], it may be preferable to use an impersonal communication style in some cases, while using a more personal communication style in other cases. Therefore, sm-bots must be aware of the type and characteristics of the actors they interact with. For example, sm-bots can learn the preferences and interests of human actors, as well as the information they decide to share, from their social networks' public profiles, and refine this knowledge overtime from the actual interactions.

- Direct vs. group messaging: Direct messaging refers to one-to-one interactions between two actors knowing their respective identities; for example, sending emails to personal email addresses or communicating via Facebook direct messages. Group messaging refers to interactions between two or more actors who do not necessarily know the identities of the other members of the group. Group messaging can be private among the group members, such as in most mailing lists and Facebook group conversations, or public such as in Twitter's tweets. Sm-bots must account for privacy aspects such as how and which information they should diffuse.

- Communication technologies usage: Different communication technologies can influence how people use them, 
particularly regarding the related synchronization. For example, a single interaction by email can be comprehensive, while the same interaction may involve a sequence of messages in the case of instant messaging.

Following the above, the sm-bot enables adaptive sociallyenhanced interactions, which may be parameterized as follows within the application:

- Conversation modality: This covers the full spectrum of interaction modalities offered by today's technologies -from a binary-encoded message to a conversational agent- and the specific realization depends on the endpoints. Considering our consultation example, the Facebook sm-bot will engage the civic actors in Facebook using a conversational social bot that is considered more user-friendly. On the other hand, the contribution of the very same civic actor to a proposal writing will lead the Facebook sm-bot to send a binary-encoded message to the GDoc sm-bot.

- Profile management: The sm-bots manage the profiles of the involved actors according to the specifics of the application but also -and as importantly- the features of the underlying platforms and the access control set by the actors. For instance, the Facebook sm-bot will have access to the Facebook profile that the end-user is willing to share; it will further autonomously enrich its knowledge based on the actions allowed by the end-user. Similarly, the GDoc sm-bot will gather knowledge about the contributing users as allowed by them.

- Interaction paradigm: Social middleware supports the traditional client-server and publish-subscribe interaction paradigms, which allows dealing with the spectrum of socially-enhanced interactions characterized above.

\section{From Vision to Civic-oriented Distributed Systems}

Our previous work on the development of the USNB solution provides us with the initial baseline solution to develop the sm-bot component enabling the creation of civic social networks. Still, enabling socially-enhanced interactions among the diverse actors -people, software services and thingscontributing to civic-oriented applications remains an open challenge. In the previous sections, we have outlined the resulting design principles for the sm-bot, and we are now investigating the following research questions toward the design and development of the actual sm-bot components:

- Sm-bot autonomous conversation manager: Sm-bots need to implement conversation with the civic actors of the related platforms. Such conversation should adapt to the context of the interaction as well as the profiles of the target actors. Designing this sm-bot capability is a longterm research challenge. We will investigate an initial design based on relevant state-of-the-art AI solutions, including existing frameworks for the management of conversational social bots.

- Sm-bot interaction protocols: The design of sm-bot interaction protocols relates to core distributed system research and development. Hence, relying on known interaction paradigms and protocols, our research focuses on their social enhancement, starting with the resulting requirements upon the communication (e.g., temporality, openness, dependability, confidentiality, security, privacy, ethics, etc.).

- Sm-bot synthesis: We are studying a Domain Specific Language associated with the core functions of the smbots, so as to ease the declarative specification of their mapping to the target platforms and further automate the synthesis of most of the sm-bots' concrete implementation.

\section{Fostering Civic Engagement}

The social middleware core enacts the actor abstraction, where entities as diverse as people, things and software, may interact and coordinate toward some civic action. As presented in the previous section, a key feature of the social middleware is to create a civic social network overlay that implements socially-enhanced interoperability across the relevant digital platforms -online social networks, civic technologies, IoT. A remaining challenge for online civic engagement is to foster the participation of people. Obviously, providing people with access to digital tools that ease their online participation to some civic action is not sufficient to ensure that people will get involved. Analysis of the related state of the art then allows us to identify some supporting functions to be offered as part of the social middleware.

\section{A. Supporting Intrinsic Motivation}

Gamification is one of the famous approaches to motivate people to participate in activities. Gamification has been extensively studied over the last few years, with literature reviews of empirical studies investigating whether and how it is an effective approach [43]. However, it is quite challenging to assess the effectiveness of gamification independently of its application domain, especially in the context of civic engagement. Still, a review like the one reported in [44] provides relevant insight, as it focuses on gamification in crowdsourcing, which is one of the main forms of e-participation. The empirical findings indicate that rankings seem to motivate users, while mixing several motivation affordances so as to target diverse groups is appealing even if not always successful. Due to the importance of participation in civic engagement, gamification design has been studied for this specific application context, e.g., [45], [16]. The review in [46] concentrated on the literature about gamification of e-participation and found that points was the most commonly employed gamification design element. The study also highlights the potential negative impact of competitive gamification, which suggests to shift the focus from competition among users to among ideas.

Although gamification is often synonymous with rewards and implemented using points, the study in [47] sheds light on the fact that there is another way to encourage behavior, that is, through building intrinsic motivation. In other words, the system should help users find their own motivation for engaging, which depends on [48]: mastery (learning something), 
autonomy, and relatedness (learning about the others who are engaged). Similar recommendations are drawn in [49]. It is our view that implementing a participation process so that the participants may build intrinsic motivation is likely the most relevant approach in the context of civic engagement.

In general, engineering gamified software is a complex process, which further requires gathering experts from various disciplines -e.g., software engineering but also psychology, design- [50]. Embedding supporting functions within the social middleware would be no exception. However, the principles of intrinsic motivation allow us to elicit the following base functions:

- Mastery: The participant must be able to acquire new knowledge in relation with the purpose of the civic action. By enabling interaction across actors, this is well supported by the social middleware core, i.e., the diverse types of actors -people, software services and things- are able to nurture each other's knowledge across time.

- Autonomy: The participants should feel that they remain in control, which primarily depends on the implementation of the upper layer application rather than the middleware functions. However, this also suggests that the participants must have the ability to set with which actors they are willing to interact as well as in which application actions they want to engage.

- Relatedness: This relates to the feeling of being part of a community -not being alone. This is well supported by the socially-enhanced interactions enabled by the social middleware core, while the civic-oriented application should foster as much as possible the virtual meeting of people as part of the civic actions they engage in. Of course, this should be implemented in a way that does not counterfeat the participant's autonomy.

\section{B. Configuring the participation process}

The actions underlying civic participation processes are well analyzed in the context of Civic Tech [25], [24]. They relate to: connecting with relevant actors, informing by giving access to relevant knowledge, contributing and acting by enabling collaboration toward joint proposal making, voting to reach decision, and ultimately implementing the outcome.

There exist various methods for involving the public in decision-making (e.g., see [51] for a survey of methods and related assessment). However, a large part of the methods enabling the extended involvement of the participants are oriented toward physical meetings of rather small, yet representative, groups of people. Our objective with the social middleware is to enable civic actions at the larger Internet scale while building upon the practices of proven participation methods. In particular, existing guides to participation have suggested key principles for the selection of methods [51]: (i) set clear objectives, (ii) identify and target all the relevant stakeholders, (iii) tailor the participation process according to the first two criteria, (iv) set out the process in an honest and understandable way, (v) ensure that the participation is timely and allow sufficient time, (vi) ensure that the process is credible, (vii) ensure that the process is interactive, (viii) ensure that the process generates a response, and (ix) only make commitments that can be kept.

Obviously, the above guidelines are primarily for use by the designers of civic actions and related civic-oriented applications, while the social middleware needs to provide the related functions that deal with the interactions and coordination of the contributing actors. This results in the following supporting framework for the customization of the sm-bots as part of a specific civic-oriented application:

- Definition of the actor base: Starting from the elicitation of the civic actors targeted by the application, customized sm-bots need to be implemented and deployed within the related platforms. Considering our running example, the platforms are: Facebook, email, GoogleDoc, monitoring networks, voting service and visualization service. The sm-bots must then initialize and maintain the base of users contributing to the application via these platforms.

- Definition of the participation workflow: Provided the civic actor and sm-bot abstractions, the implementation of the civic-oriented application relies on customizing the sm-bots with respect to coordinating the involved civic actors. The customization then subdivides into implementing: (i) the interaction processes with the inner civic actors and (ii) the coordination processes among the distributed sm-bots.

- Customize the interaction with participating actors: As part of the above, the interaction processes with the inner civic actors relate to the implementation of the specialized socially-enhanced interactions, as presented in Section IV-B.

\section{Trust, security \& privacy}

Social middleware must ensure that the participation process that it enables guarantee security and privacy to the involved participants. Further, given the openness of the system, trust management must be implemented to ensure that all the participants behave properly. The literature is rich of technology solutions to manage trust, security and privacy. Regarding privacy enforcement, we may in particular leverage the solutions dedicated to crowdsourcing, e.g., [52], [53]. We will thus integrate state of the art solutions to trust, security and privacy within the social middleware.

A related concern that arises in the context of civic engagement follows from the vicious trends of fake news, privacy leakages, surveillance, and cyberwarfare [34], [54], [55], [56]. Given social middleware, where everything -no matter its nature- can interact in the virtual world, and where data flow across multiple independent and heterogeneous systems, it is imperative to acknowledge such trends and to devise mechanisms to fight them. Indeed, social middleware can be an attractive environment for deploying tools of mass surveillance and manipulation, or for taking advantage of the large amounts of available data for diverse purposes.

We highlight two aspects of social middleware for which contributing developers must take special care: 
1) Sensitive information flowing across the boundaries of multiple systems. Consider, for example, a Facebook user sending a private message to a Twitter user or civic tech platform through social middleware. This message can be intercepted, compromised or transformed while flowing between actors and systems. Moreover, privacy policies may vary across systems, highlighting the need for modeling privacy aspects and understanding its consequences at the social middleware level. The civic engagement functions of the sm-bot related to privacy and security serve that purpose. Still, the specification of policies that the sm-bots enforce remains under the responsibility of developers.

2) The deployed instances of sm-bots can be hacked. In particular, the functions dedicated to the enhancement of social interaction may be an interesting target since they interact directly with humans. For example, a social bot launched by the sm-bot may start impersonating any connected actor of the cyber-physical layer and then spread deceptive information.

There exist tools for bot detection [57], which allow blocking their interaction. However, sm-bots are the main component of social middleware to assist humans toward civic engagement, informing them about what is happening in the physical world, and enabling them to contribute and act. Knowing that an actor is not human -as state of the art bot detection does- is not enough; researchers must continue working on the detection of deceptive information and malicious behavior, regardless of whether or not actors are human.

On a complementary note, being the target of cyber-attacks is an inevitable risk for any digital system, especially for open distributed systems that are socially-oriented as social middleware is. Systems must be and are increasingly designed with that risk in mind. Further, we believe that social middleware will empower collective actions and citizens will be able to build new governance institutions supported by the cyberphysical convergence provided by such middleware. Additionally, social middleware allows the verification of facts across multiple sources -humans, connected objects, and software platforms- from a variety of distributed and heterogeneous systems.

\section{CONCLUSION}

This paper puts forward the vision of social middleware as an essential element to support civic engagement at scale in today's digital era. Social middleware enables civic engagement ecosystems composed of diverse actors -human citizens, connected objects, and software services- existing in heterogeneous digital interaction environments -social media and connected object/service platforms. The ultimate purpose is to enhance the quality of participation by: (i) allowing citizens to use their favorite communication technologies to participate; (ii) allowing citizens to interact not only with other citizens but also with connected objects and software services so as to get access to relevant value-added knowledge; and (iii) leveraging Civic Tech as well as enhancing its capabilities to foster the engagement of people.

Our ongoing and future work focuses on the further development of sm-bots for a concrete civic engagement application to assess the early design of the social middleware building blocks. This development will inform the subsequent development of a method and tools to support the synthesis of sm-bots based on the declarative specification of the functions provided by third-party platforms hosting the (civic) actors.

As part of the above, we are investigating the various incentive mechanisms that we can implement within social middleware by taking advantage of the different functionalities offered by social media. Related to that, looking into anthropomorphism for civic engagement may be a way to go, as in [58] where authors highlight the relevance of anthropomorphism and social presence to important business-related outcomes, such as attitudes, satisfaction and the emotional connection that consumers feel with a company after interacting with social bots. How much more human-like sm-bots can improve the quality of civic engagement is the underlying research question.

It is also crucial to continuously investigate the impact of disruptive innovations on the civic technology ecosystem and hence, how these innovations can influence social middleware. For example, social middleware may leverage blockchain-based solutions for digital identities such as ID2020 (https://id2020.org) whose goal is to provide internationally-recognized digital identities, particularly for populations whose governments provide weak identity systems or whose identities are at risk for political reasons.

Concluding, social middleware, as presented here, poses the vision of a middleware solution contributing to civic engagement. The approach then raises the question of its midto-long term impact on civic participation processes in relation with the level of enacted automation. For instance, "Can proactive social bots play an active role in participatory processes within social middleware?" Obviously, critical actions of participatory processes -e.g., voting- are restricted to human participants only. However, some actions may benefit from the intervention of non-human participants based on the knowledge about the supported process, to instigate engagement. For example, in the context of proposal making, bots may submit ideas, or hints to ideas, so that human participants can decide if they are worth it and, if they are, they can develop them. Bots may also discover relevant digital resources/data about the ongoing process, which they may expose to the contributing actors.

Finally, we stress the trans-disciplinary nature of digital tools for civic engagement. It is essential that computer scientists, human and social scientists, engineers, citizens and government representatives collaborate for the development of such tools, from design to implementation to actual use.

\section{REFERENCES}

[1] H. Kukka, M. Foth, and A. K. Dey, "Transdisciplinary approaches to urban computing," Int. J. Hum.-Comput. Stud., vol. 81, no. C, Sep. 2015. 
[2] M. Tsvetkova, T. Yasseri, E. T. Meyer, J. B. Pickering, V. Engen, P. Walland, M. Lüders, A. Følstad, and G. Bravos, "Understanding human-machine networks: A cross-disciplinary survey," ACM Comput. Surv., vol. 50, no. 1, Apr. 2017.

[3] A. Gupta, A. Kalra, D. Boston, and C. Borcea, "Mobisoc: A middleware for mobile social computing applications," Mob. Netw. Appl., vol. 14, no. 1, Feb. 2009.

[4] L. Chen, K. Chen, C. Shao, and P. Zhu, "Socaware: A middleware for social applications in online social networks," in 13th IEEE International Conference on Trust, Security and Privacy in Computing and Communications (TrustCom), 2014

[5] A. Mehrotra, V. Pejovic, and M. Musolesi, "Sensocial: A middleware for integrating online social networks and mobile sensing data streams," in Proceedings of the 15th International Middleware Conference, 2014.

[6] R. Baden, A. Bender, N. Spring, B. Bhattacharjee, and D. Starin, "Persona: An online social network with user-defined privacy," in Proceedings of the ACM SIGCOMM Conference on Data Communication, 2009.

[7] P. Alves and F. Paulo, "Anonylikes: Anonymous quantitative feedback on social networks," in Proceedings of the 14th International Middleware Conference, 2013

[8] D. Koll, J. Li, and X. Fu, "Soup: An online social network by the people, for the people," in Proceedings of the 15th International Middleware Conference, 2014

[9] U. Lehner, M. Baldauf, V. Eranti, W. Reitberger, and P. Fröhlich, "Civic engagement meets pervasive gaming: Towards long-term mobile participation," in CHI Extended Abstracts on Human Factors in Computing Systems, 2014

[10] M. Harding, B. Knowles, N. Davies, and M. Rouncefield, "HCI, civic engagement \& trust," in Proceedings of the 33rd Annual ACM Conference on Human Factors in Computing Systems, 2015.

[11] J. Kim, E.-Y. Ko, J. Jung, C. W. Lee, N. W. Kim, and J. Kim, "Factful: Engaging taxpayers in the public discussion of a government budget," in Proceedings of the 33rd Annual ACM Conference on Human Factors in Computing Systems, 2015.

[12] F. Salim and U. Haque, "Urban computing in the wild: A survey on large scale participation and citizen engagement with ubiquitous computing, cyber physical systems, and internet of things," International Journal of Human - Computer Studies, vol. 81, pp. 31-48, 2015.

[13] K. Boehner and C. DiSalvo, "Data, design and civics: An exploratory study of civic tech," in Proceedings of the CHI Conference on Human Factors in Computing Systems, 2016.

[14] L. Carton and P. Ache, "Citizen-sensor-networks to confront government decision-makers: Two lessons from the netherlands," Journal of Environmental Management, vol. 196, 2017.

[15] A. May and T. Ross, "The design of civic technology: factors that influence public participation and impact," Ergonomics, vol. 61, no. 2, 2018.

[16] M. V. Palacin-Silva, A. Knutas, M. A. Ferrario, J. Porras, J. Ikonen, and $\mathrm{C}$. Chea, "The role of gamification in participatory environmental sensing: A study in the wild," in Proceedings of the CHI Conference on Human Factors in Computing Systems, 2018.

[17] M. Conti, A. Passarella, and S. K. Das, "The internet of people (iop): A new wave in pervasive mobile computing," Pervasive and Mobile Computing, vol. 41, pp. 1 - 27, 2017. [Online]. Available: http://www.sciencedirect.com/science/article/pii/S1574119217303723

[18] T. Dubow, A. Devaux, C. V. Stolk, and C. Manville, "Civic engagement: How can digital technologies underpin citizen-powered democracy?," RAND Corporation and Corsham Institute, Tech. Rep., 2017. [Online]. Available: https://www.rand.org/pubs/conf_proceedings/CF373.html

[19] P. Dahlgren, Media and political engagement. Cambridge: Cambridge University Press, 2009.

[20] T. Poell and J. Van Dijck, "Social media and activist communication," The Routledge Companion to Alternative and Community Media, 2015.

[21] A. Kavada, "Creating the collective: social media, the occupy movement and its constitution as a collective actor," Information, Communication \& Society, vol. 18, no. 8, 2015.

[22] A. Dale and A. Strauss, "Don't forget to vote: text message reminders as a mobilization tool," American Journal of Political Science, vol. 53 , no. $4,2009$.

[23] R. Angarita, N. Georgantas, and V. Issarny, "USNB: Enabling universal online social interactions," in IEEE 3rd International Conference on Collaboration and Internet Computing (CIC), 2017.
[24] R. Ghorayeb and M. Soule, "Can Civic Tech Save Democracy? How Technology is Renewing Civic Engagement," L'Atelier BNP Paribas North America, Tech. Rep., 2017. [Online]. Available: https://atelier. bnpparibas/en/smart-city/article/study-civic-tech-save-democracy

[25] J. Holston, V. Issarny, and C. Parra, "Engineering Software Assemblies for Participatory Democracy: The Participatory Budgeting Use Case," in Proceedings of the 38th International Conference on Software Engineering: Software Engineering in Society Track. ACM, 2016.

[26] C. Parra, C. Rohaut, M. Maeckelbergh, V. Issarny, and J. Holston, "Expanding the Design Space of ICT for Participatory Budgeting," in Communities and Technologies, 2017. [Online]. Available: https: //hal.inria.fr/hal-01519127

[27] A. S. Taylor, S. Lindley, T. Regan, D. Sweeney, V. Vlachokyriakos, L. Grainger, and J. Lingel, "Data-in-place: Thinking through the relations between data and community," in Proceedings of the 33rd Annual ACM Conference on Human Factors in Computing Systems, 2015.

[28] M. Balestrini, T. Diez, P. Marshall, A. Gluhak, and Y. Rogers, "IoT community technologies: leaving users to their own devices or orchestration of engagement?" EAI Endorsed Transactions on Internet of Things, vol. 1 , no. 1,2015

[29] V. Issarny, V. Mallet, K. Nguyen, P.-G. Raverdy, F. Rebhi, and R. Ventura, "Dos and don'ts in mobile phone sensing middleware: Learning from a large-scale experiment," in Proceedings of the 17th International Middleware Conference. ACM, 2016.

[30] B. Lefèvre and V. Issarny, "Matching Technological \& Societal Innovations: The Social Design of a Mobile Collaborative App for Urban Noise Monitoring," in 4th IEEE International Conference on Smart Computing, 2018. [Online]. Available: https://hal.inria.fr/hal-01801314

[31] A. Sheth and P. Anantharam, "Physical cyber social computing for human experience," in Proceedings of the 3rd International Conference on Web Intelligence, Mining and Semantics, 2013.

[32] J. Zeng, L. Yang, M. Lin, H. Ning, and J. Ma, "A survey: Cyber-physicalsocial systems and their system-level design methodology," 2016.

[33] E. Ferrara, O. Varol, C. Davis, F. Menczer, and A. Flammini, "The rise of social bots," Commun. ACM, vol. 59, no. 7, 2016.

[34] C. Shao, G. L. Ciampaglia, O. Varol, A. Flammini, and F. Menczer, "The spread of fake news by social bots," arXiv preprint arXiv:1707.07592, 2017.

[35] A. Antonin, L. Lup, G. Boella, S. Buccoliero, and C. Schifanella, "Collaborative Multi-Perspective Urban Knowledge and Civic media," in 6th International Conference on Advanced Collaborative Networks, Systems and Applications, 2016.

[36] B. Latour, Reassembling the social: An introduction to actor-networktheory. Oxford university press, 2005.

[37] R. Angarita, N. Georgantas, C. Parra, J. Holston, and V. Issarny, "Leveraging the service bus paradigm for computer-mediated social communication interoperability," in Proceedings of the 39th International Conference on Software Engineering: Software Engineering in Society Track, ser. ICSE-SEIS '17. IEEE Press, 2017, pp. 67-75. [Online]. Available: https://doi.org/10.1109/ICSE-SEIS.2017.9

[38] R. Angarita, B. Lefevre, S. Ahvar, E. Ahvar, N. Georgantas, and V. Issarny, "Universal social network bus: Towards the federation of heterogeneous online social network services," 2019, submitted for publication - Available upon request to the authors.

[39] M. Lippi, M. Mamei, S. Mariani, and F. Zambonelli, "Coordinating distributed speaking objects," in IEEE 37th International Conference on Distributed Computing Systems (ICDCS), 2017.

[40] R. E. Petty and J. T. Cacioppo, "The elaboration likelihood model of persuasion," in Communication and persuasion. Springer, 1986.

[41] J. B. Walther, "Computer-mediated communication: Impersonal, interpersonal, and hyperpersonal interaction," Communication research, vol. 23 , no. $1,1996$.

[42] — "Theories of computer-mediated communication and interpersonal relations," The handbook of interpersonal communication, vol. 4, 2011

[43] J. Hamari, J. Koivisto, and H. Sarsa, "Does gamification work? - a literature review of empirical studies on gamification," in 47th Hawaii International Conference on System Sciences (HICSS), 2014. [Online]. Available: doi.ieeecomputersociety.org/10.1109/HICSS.2014.377

[44] B. Morschheuser, J. Hamari, and J. Koivisto, "Gamification in crowdsourcing: A review," in 49th Hawaii International Conference on System Sciences (HICSS), 2016.

[45] S. Rehm, M. Foth, and P. Mitchell, "Dogood: examining gamification, civic engagement, and collective intelligence," AI \& SOCIETY, vol. 33, no. 1,2018 . 
[46] L. Hassan and J. Hamari, "Gamification of e-participation: A literature review," in 52nd Hawaii International Conference on System Sciences(HICSS), 2019. [Online]. Available: doi.ieeecomputersociety. org/10.1109/HICSS.2014.377

[47] S. Nicholson, A RECIPE for Meaningful Gamification. Springer International Publishing, 2015. [Online]. Available: https://doi.org/10. 1007/978-3-319-10208-5_1

[48] E. Deci and R. Ryan, Handbook of Self-Determintation Research. University of Rochester Press, 2004.

[49] A. Suh, C. Wagner, and L. Liu, "Enhancing user engagement through gamification," Journal of Computer Information Systems, vol. 58 no. 3, 2018. [Online]. Available: https://doi.org/10.1080/08874417. 2016.1229143

[50] B. Morschheuser, L. Hassan, K. Werder, and J. Hamari, "How to design gamification? a method for engineering gamified software," Information and Software Technology, vol. 95, 2018. [Online]. Available: http://www.sciencedirect.com/science/article/pii/S095058491730349X

[51] J. Petts and B. Leach, "Evaluating methods for public participation: Literature review," The University of Birmingham, Tech. Rep., 2000.

[52] H. Kajino, H. Arai, and H. Kashima, "Preserving worker privacy in crowdsourcing," Data Min. Knowl. Discov., vol. 28, no. 5-6, 2014. [Online]. Available: http://dx.doi.org/10.1007/s10618-014-0352-3

[53] L. Béziaud, T. Allard, and D. Gross-Amblard, "Lightweight PrivacyPreserving Task Assignment in Skill-Aware Crowdsourcing," 2017. [Online]. Available: https://hal.inria.fr/hal-01534682

[54] C. Cadwalladr, "Google, democracy and the truth about internet search," The Guardian, vol. 4, no. 12, 2016.

[55] H. Allcott and M. Gentzkow, "Social media and fake news in the 2016 election," Journal of Economic Perspectives, vol. 31, no. 2, 2017.

[56] S. C. Woolley, "Automating power: Social bot interference in global politics," First Monday, vol. 21, no. 4, 2016.

[57] N. Hassan et al., "Toward automated fact-checking: Detecting checkworthy factual claims by claimbuster," in Proceedings of the $23 \mathrm{rd}$ ACM SIGKDD International Conference on Knowledge Discovery and Data Mining. ACM, 2017.

[58] T. Araujo, "Living up to the chatbot hype: The influence of anthropomorphic design cues and communicative agency framing on conversational agent and company perceptions," Computers in Human Behavior, vol. 85,2018 